\title{
The 45th Annual Meeting of the European Society for Blood and Marrow Transplantation: Working Parties
}

C Springer Nature Limited 2019

\section{4-27 March 2019 Frankfurt, Germany}

Modified and published with permission from https://www.ebmt.org/annual-meeting

Sponsorship Statement: Publication of this supplement is sponsored by the European Society for Blood and Marrow Transplantation. All content was reviewed and approved by the EBMT Committee, which held full responsibility for the abstract selections.

\section{WP01}

Next generation sequencing (NGS) in acute myeloid leukaemia (AML): An update

\section{Torsten Haferlach ${ }^{1}$}

${ }^{1}$ MLL Munich Leukemia Laboratory, Munich, Germany

Background: The landscape of AML, with respect to cytogenetics and especially molecular genetics, dramatically increased over the last 15 years. This has to be acknowledged for diagnosis, classification (WHO), prognostication and for measurable residual disease (MRD).

Methods: Next generation sequencing (NGS) provided most of the recent findings in the biology of AML. High throughput sequencing including even whole genome sequencing, whole exome sequencing and RNA-seq led to new data on biology, characterisation of the respective AML, MRD markers and also new targets for drug development and precision medicine approaches. Turnaround times are decreasing to less than a week, making it even an important tool at diagnosis of AML.

Results: Due to parallel sequencing of many samples in one NGS run, short turnaround times for wet lab assays and sequencing itself and especially fostered by new bioinformatics approaches the NGS can be applied in clinical routine. This is true for diagnosis as well as for follow-up, also an increasing number of assays for MRD have been shown to be useful. The broad spectrum of findings and their clinical relevance and individual power for decision making makes it quite difficult today to use. There is no doubt that genes such as NPM1, CEBPA, FLT3, TP53, ASXL1 and $R U N X 1$ as well as chromosomal changes, described by WHO classification, have diagnostic and clinical relevance. For other genes, such as DNMP3A and TET2, clinical means have to be defined for the individual patient. Any treatment decision based on the cytogenetic and molecular genetic background of AML has to be more individualised today.

Conclusions: NGS now opens the field of a comprehensive cytogenetic and especially molecular genetic description of the individual AML. This is challenging for any clinical decision at diagnosis, for prognostication and also for follow-up including MRD. This is also of increasing importance for any kind of precision medicine and individualised treatment options. NGS will be the tool for the next years to support all these important goals.

Disclosure: None.

\section{WP02}

Abstract already published

WP03

Abstract already published. 


\section{WP04}

Pitfalls in prediction model development and validation in small data sets

\section{Ewout W. Steyerberg ${ }^{I}$}

${ }^{1}$ Leiden University Medical Center, Leiden, Netherlands

Background: Prediction models are often based on relatively small datasets. Small data sets pose particular problems with model specification, estimation, and validation. In this presentation I aim to illustrate some of the problems and their potential solutions.

Methods: These problems include the instability of standard but suboptimal model building strategies such as stepwise selection of candidate predictors for regression models, and the optimism in model performance when developed from a small sample. Potential remedies come from modern penalized estimation approaches.

Results: I will contrast approaches in small, carefully registered datasets, with new paradigms of Big Data and Machine Learning techniques.

Conclusions: The importance of validation of prediction models through a range of settings will be emphasized.

Disclosure: None.

\section{WP05}

Developing and assessing prediction models for survival outcomes based on data with missing observations

Liesbeth C. de Wreede ${ }^{1,2}$, Hein Putter ${ }^{1}$, Johannes Schetelig $^{2,3}$, Bart J.A. Mertens ${ }^{1}$

${ }^{1}$ LUMC, Leiden, Netherlands, ${ }^{2}$ DKMS Clinical Trials Unit, Dresden, Germany, ${ }^{3}$ University Hospital Dresden, Dresden, Germany

Background: Prediction models are often based on datasets with missing observations for some of the predictors.
Especially in the context of registry data, this is a common problem. Ignoring these observations leads to a loss of information and might also cause bias.

Methods: A common method to deal with missing data is multiple imputation. Although there are rules for estimating regression coefficients in this setting, there is no standard approach for calculating predicted probabilities. An extra complication occurs when the outcomes are survival outcomes, in which context the event of interest will not be observed for all patients (censored observations). The most commonly used prediction model in this context is a Cox model, where we have to consider both the baseline hazard and the regression coefficients if we want to predict survival outcomes at certain timepoints, e.g., overall survival at 1 or 5 years after transplantation.

Results: We present different methods to derive these predictions for individual patients by pooling parameters estimated in imputed datasets, and show results, in particular with respect to variability and bias in predicted outcomes. We also explain how the predictive performance of models can be assessed when no external validation dataset is available, taking into account the missing values. To avoid positive bias by evaluation in the dataset on which the model was developed, we present an implementation which intertwines cross-validation and multiple imputation.

Conclusions: All methods and issues are illustrated on an EBMT dataset to demonstrate their relevance. The characteristics of the different approaches are further explored by means of simulated data which enable a systematic study of the impact of different missingness patterns, number of multiply imputed datasets and strength of association between the predictor with missing values and the outcome. Implications for practice and aspects needing further research are discussed.

Disclosure: None.

\section{WP06}

Abstract already published. 